\title{
Response to the letter by F. Hadziselimovic
}

\author{
Ricardo González • Barbara Ludwikowski
}

Received: 3 April 2012 / Accepted: 4 April 2012 /Published online: 26 April 2012

(C) Springer-Verlag 2012

Sir, we thank Professor Hadziselimovic for his interest in our paper and the comments he made. He raised the alarming scenario of azoospermia developing in males with former cryptorchidism. The paper by Fedder [1] reported on a series of 100 men with azoospermia who consulted a specialized clinic in Denmark. Of them, 27 had a history of undescended testis (UDT). No causal relationship between the two conditions was established. These findings are impossible to interpret from an epidemiologic perspective. The study population was likely to be highly selected, and it is not stated what proportion had bilateral UDT. Also it is unclear whether or not they had received hormonal treatment or if the contralateral testes had been biopsied. Although a minor procedure, testicular biopsy incurs the risk of epididymal injury and therefore we discourage its routine use.

Fogle et al. in a report of a population of azoospermic men in California found that only 1 of 55 men investigated had a history of UDT [2]. Furthermore, the incidence of azoospermia in subfertile men populations varied with ethnicity and geography between 3 and $20 \%$. Hence, the data reported by Fedder [1], however interesting, can hardly be taken as representative of formerly cryptorchid men in the general population. We emphasize that in nonazoospermic men, there is a poor correlation between semen parameters and paternity, making prediction of fertility difficult $[3,4]$.

R. González $(\bowtie) \cdot$ B. Ludwikowski

Kinderkrankenhaus auf der Bult,

Hannover, Lower Saxony, Germany

e-mail: ricardo_gonzalez33154@yahoo.com
The other issues raised in the letter are addressed in detail in the text of our article. Notwithstanding the objections raised and the data reported in reference 3 of the letter, the fact remains that paternity potential for men with a history of unilateral UDT is normal, and in the absence of good evidence (based on prospective randomized controlled studies) of the benefits of hormonal treatment (pre- or postoperative) looking at fertility (not fertility potential) as the end point, we stand by our conclusions. If countries like Germany, where existing guidelines recommend hormonal treatment for unilateral UDT, abandoned its routine use, at least 180 euros (medication alone, not including professional services) would be saved for each of $1 \%$ of the boys born in a particular year (about $680,000 €$ in 2010).

\section{References}

1. Fedder J (2011) History of cryptorchidism and ejaculate volume as simple predictors for the presence of testicular sperm. Syst Biol Reprod Med 57(3):154-161

2. Fogle RH, Steiner AZ, Marshall FE, Sokol RZ (2008) Etiology of azoospermia in a large nonreferral inner-city population. Fertil Steril 86(1):197-199

3. Niederberger C, Joyce G, Wise M and Meacham RB (2007) Male infertility. In Litwin M, Saigal C (eds) Urologic diseases in America. US Department of Health and Human Services, Public Health Service, National Institutes of Health, National Institute of Diabetes and Digestive and Kidney Diseases. Washington, DC: US Government Printing Office; NIH Publication No. 07-5512, pp. $461-481$

4. Sripada S, Townend J, Campbell D, Murdoch L, Mathers E, Bhattacharya S (2010) Relationship between semen parameters and spontaneous pregnancy. Fertil Steril 94(2):624-630 